\title{
Sex differences in the phylum-level human gut microbiota composition
}

\author{
Alexander Koliada', Vladislav Moseiko', Mariana Romanenko², Oleh Lushchak ${ }^{3,4}$, Nadiia Kryzhanovska ${ }^{5}$, \\ Vitaly Guryanov ${ }^{6}$ and Alexander Vaiserman ${ }^{2 *}$
}

\begin{abstract}
Background: Evidence was previously provided for sex-related differences in the human gut microbiota composition, and sex-specific discrepancy in hormonal profiles was proposed as a main determinant of these differences. On the basis of these findings, the assumption was made on the role of microbiota in the sexual dimorphism of human diseases. To date, sex differences in fecal microbiota were demonstrated primarily at lower taxonomic levels, whereas phylum-level differences between sexes were reported in few studies only. In the present population-based cross-sectional research, sex differences in the phylum-level human gut microbiota composition were identified in a large (total $n=2301$ ) sample of relatively healthy individuals from Ukraine.

Results: Relative abundances of Firmicutes and Actinobacteria, as determined by qRT-PCR, were found to be significantly increased, while that of Bacteroidetes was significantly decreased in females compared to males. The Firmicutes to Bacteroidetes (F/B) ratio was significantly increased in females compared to males. Females had $31 \%$ higher odds of having F/B ratio more than 1 than males. This trend was evident in all age groups. The difference between sexes was even more pronounced in the elder individuals (50+): in this age group, female participants had $56 \%$ higher odds of having F/B ratio $>1$ than the male ones.
\end{abstract}

Conclusions: In conclusion, sex-specific differences in the phylum-level intestinal microbiota composition were observed in the Ukraine population. The F/B ratio was significantly increased in females compared to males. Further investigation is needed to draw strong conclusions regarding the mechanistic basis for sex-specific differences in the gut microbiota composition and regarding the role of these differences in the initiation and progression of human chronic diseases.

Keywords: Gut microbiota composition, Firmicutes to Bacteroidetes ratio, Sex-specific differences, Hormonal profile

\section{Background}

A wide range of microorganisms inhabit various sites of the human body, such as the skin, oral cavity and vagina, but most of them reside in the gut. Convincing evidence indicates that composition of the bacterial community inhabiting the gastrointestinal tract (gut microbiota) contributes significantly to host metabolic and immune functions, thereby substantially affecting its health status

\footnotetext{
* Correspondence: vaiserman23@gmail.com

${ }^{2}$ Institute of Gerontology, Vyshgorodskaya st. 67, 04114 Kyiv, Ukraine

Full list of author information is available at the end of the article
}

$[1,2]$. The human intestinal microbiome (the genetic material of all microorganisms, including bacteria and also some viruses and fungi, that colonize the intestine) is known to be established early in life and remains relatively stable during adult life, but differs between individuals depending on genotype, body mass index (BMI), lifestyle, physical activity, and also dietary and cultural habits [3]. Multiple findings from animal and human studies indicate that sex may also be a potentially important factor in determining the microbiome composition (for review, see ref. [4]). However, it is often ignored by researchers even despite its potential importance. In

(C) The Author(s). 2021 Open Access This article is licensed under a Creative Commons Attribution 4.0 International License, which permits use, sharing, adaptation, distribution and reproduction in any medium or format, as long as you give appropriate credit to the original author(s) and the source, provide a link to the Creative Commons licence, and indicate if changes were made. The images or other third party material in this article are included in the article's Creative Commons licence, unless indicated otherwise in a credit line to the material. If material is not included in the article's Creative Commons licence and your intended use is not permitted by statutory regulation or exceeds the permitted use, you will need to obtain permission directly from the copyright holder. To view a copy of this licence, visit http://creativecommons.org/licenses/by/4.0/ The Creative Commons Public Domain Dedication waiver (http://creativecommons.org/publicdomain/zero/1.0/) applies to the data made available in this article, unless otherwise stated in a credit line to the data. 
several recent studies, evidence for sex-related differences in the composition of intestinal microbiome was shown, and sex-specific discrepancy in hormonal profiles was proposed to be a major determinant of these differences [5]. The host and microbiota communicate in a bidirectional manner, affecting each other's functions. In particular, gut microbiota plays a key role in maintaining normal testosterone levels, estrous cycle, and reproductive functions [6]. Moreover, intestinal microorganisms have been shown to be involved in enterohepatic recirculation of estrogens and androgens, as well as in affecting local and systemic levels of sex steroid hormones and in generating androgens from glucocorticoids [6]. Furthermore, the potential role of microbiota in shaping sexually dimorphic immunity was suggested [7]. These sex-specific differences in microbiota composition can likely contribute to sexrelated distinction in local gastrointestinal inflammation, systemic immune responses and susceptibility to inflammatory disorders [8]. In addition, the gut microbiota has been repeatedly shown to be an important causal factor in pathogenesis of cardio-metabolic disorders, such as impaired glucose regulation, atherosclerosis, hypertension, dyslipidemia, obesity and type 2 diabetes $[9,10]$ as well as neurological disorders [11], for which sexual dimorphism in disease onset and progression has been consistently reported. Moreover, changes in the gut microbiome can likely contribute to the higher prevalence of autoimmune diseases in women than in men [12] and to health concerns in menopausal women [13]. The assumption on the potential role of gut microbiota in the sexual dimorphism of human diseases, however, remains mostly hypothetical. To date, the meaningful empirical evidence for sexspecific differences in the intestinal microbiota composition was reported mostly in animal models, while findings from human populations are rather inconsistent and inconclusive perhaps due to the many confounding factors involved [4]. In addition, most of this evidence comes from small sample size studies which does not allow for causal inference. Therefore, further research is needed in order to better understand sex-related differences in the composition of human intestinal microbiome. In present cross-sectional study, sex differences in the phylum-level human gut microbiota composition were identified in relatively healthy individuals from Ukrainian population. In contrast to previous research on this topic, the present study was realized with a large-size, population-based design.

\section{Results}

The differences in the gut microbial composition were taxonomically evaluated at the phylum level. The relative abundances of major gut microbiota phyla in male and female study participants across age groups are presented in Fig. 1. From this figure, it is seen that these relative abundances were quite similar across age groups in both sexes.

Nevertheless, at the level of aggregated data, statistically significant differences in the phylum-level intestinal microbial composition have been observed among sexes. More specifically, relative abundances of Firmicutes and Actinobacteria were found to be significantly increased, while that of Bacteroidetes was significantly decreased in females compared to males (Fig. 2, Supplementary Table S1). Significant differences between sexes were also observed for the Firmicutes to Bacteroidetes (F/B) ratio; this ratio was significantly increased in females compared to males.

These trends were evident in all ages. Relative abundances of Firmicutes and Actinobacteria were higher,
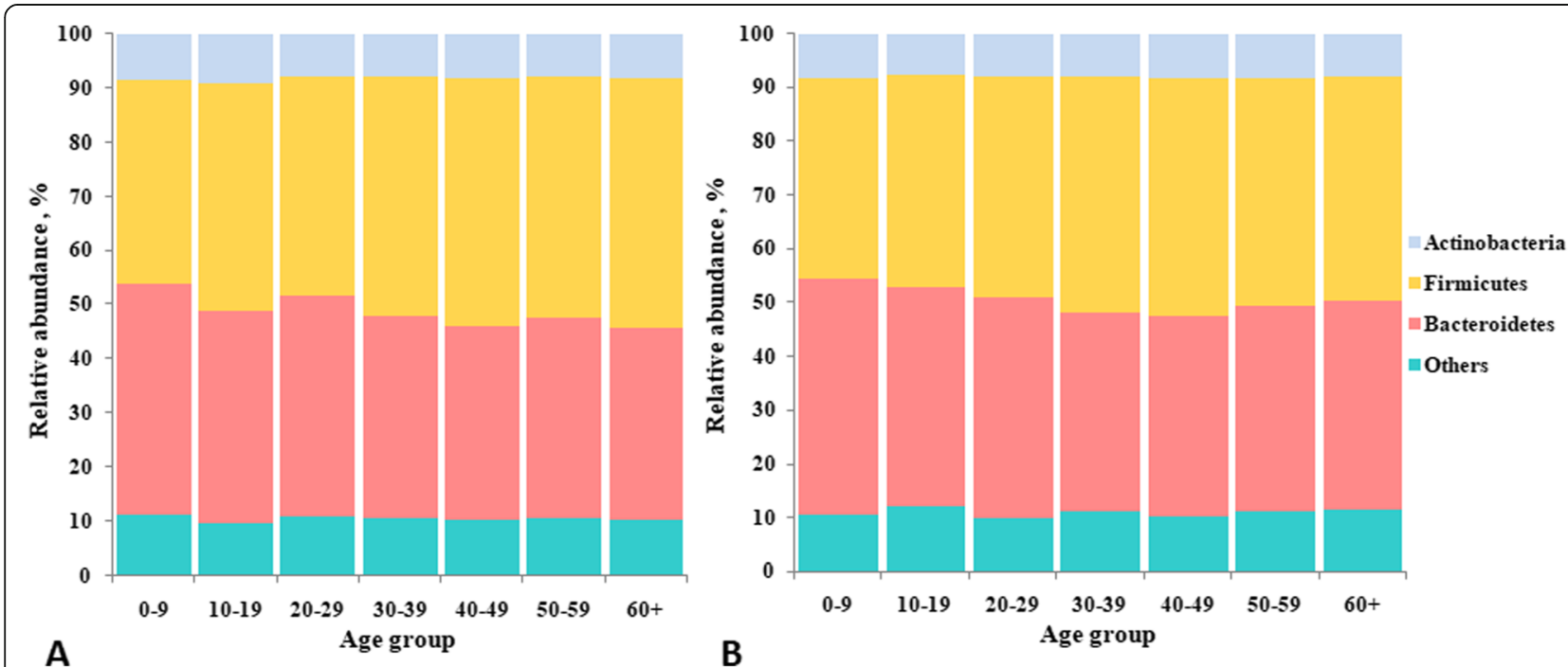

Fig. 1 Changes in relative abundance of major gut microbiota phyla in male and female study participants across age groups: a Female; b Male 

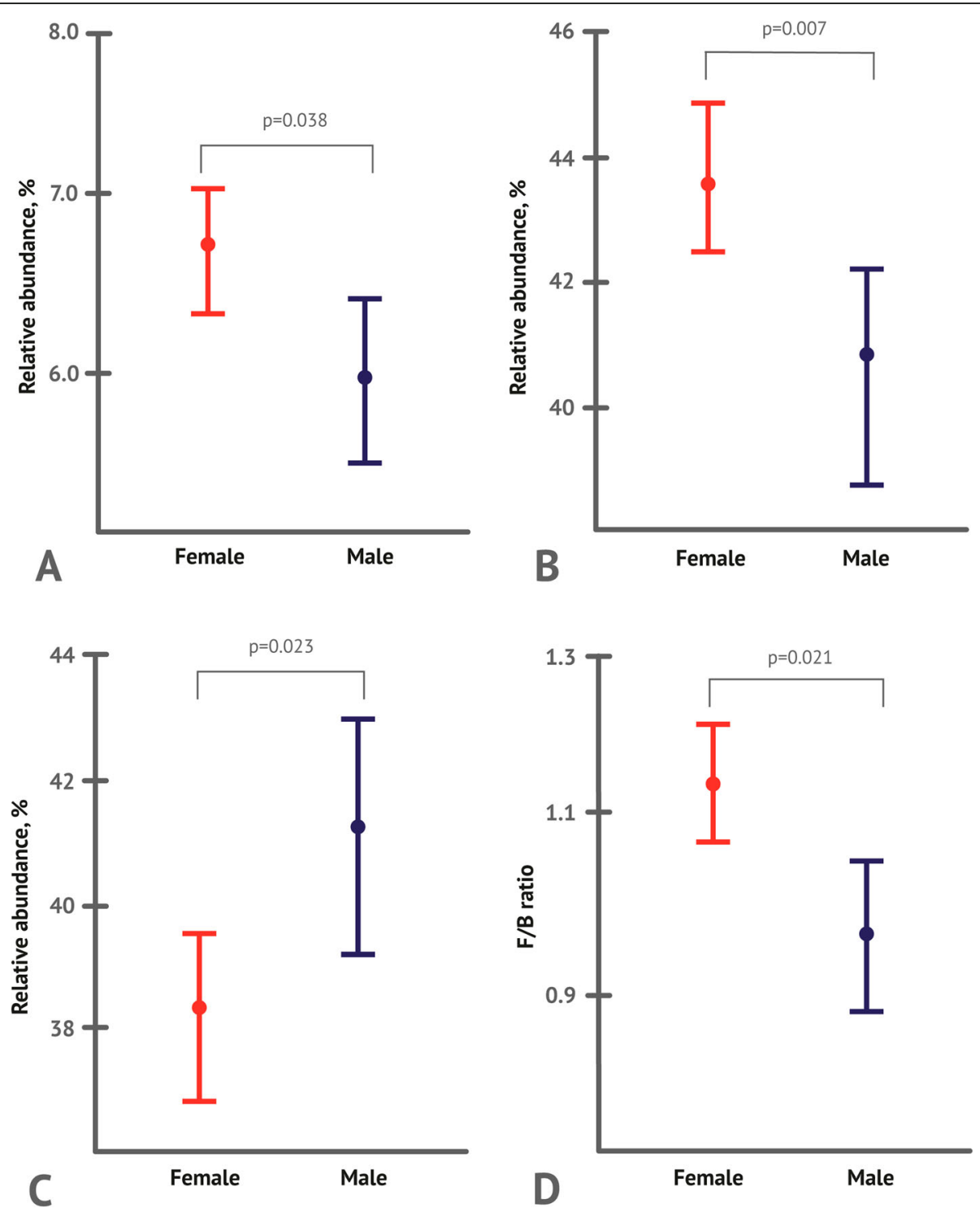

Fig. 2 Relative abundances of major gut microbiota phyla in female $(N=1515)$ and male $(N=786)$ participants: a Firmicutes, b Bacteroidetes, c Actinobacteria, and $\mathbf{d}$ F/B ratio. Data are given as median values (circles) with whiskers indicating $95 \%$ confidence intervals (Cls)

while those of Bacteroidetes were lower in female participants compared to male ones in all age groups studied (Fig. 3a-d). For all these bacterial phyla as well as for F/B ratio, effects of sex and sex $\times$ age interactions were significant according to the two-way ANOVA (Table 1).

Further, the effect of sex on F/B ratio was evaluated using logistic regression analysis. According to this analysis, females had $31 \%$ higher odds of having F/B ratio more than 1 than males (Table 2). Trend to an increased $\mathrm{F} / \mathrm{B}$ ratio in females was evident across age groups, and this sex difference was found to be most pronounced in the older age group (50+) (Fig. 3e). In this age group, female participants had $56 \%$ higher odds of having $\mathrm{F} / \mathrm{B}$ ratio $>1$ than the male ones.
In contrast to the phylum-level effects, no differences in microbiota composition were observed between sexes at both the genus and species taxonomic levels and for both intestinal normal flora and conditionally pathogenic flora (Table 3).

\section{Discussion}

The main finding of this study is that there are sexspecific differences in the phylum-level gut microbiota composition in individuals from the Ukraine population. In other human populations, sex differences in fecal microbiota were demonstrated primarily at lower taxonomic levels [4]. For example, Ding and Schloss using the Dirichlet multinomial mixture (DMM)-based 

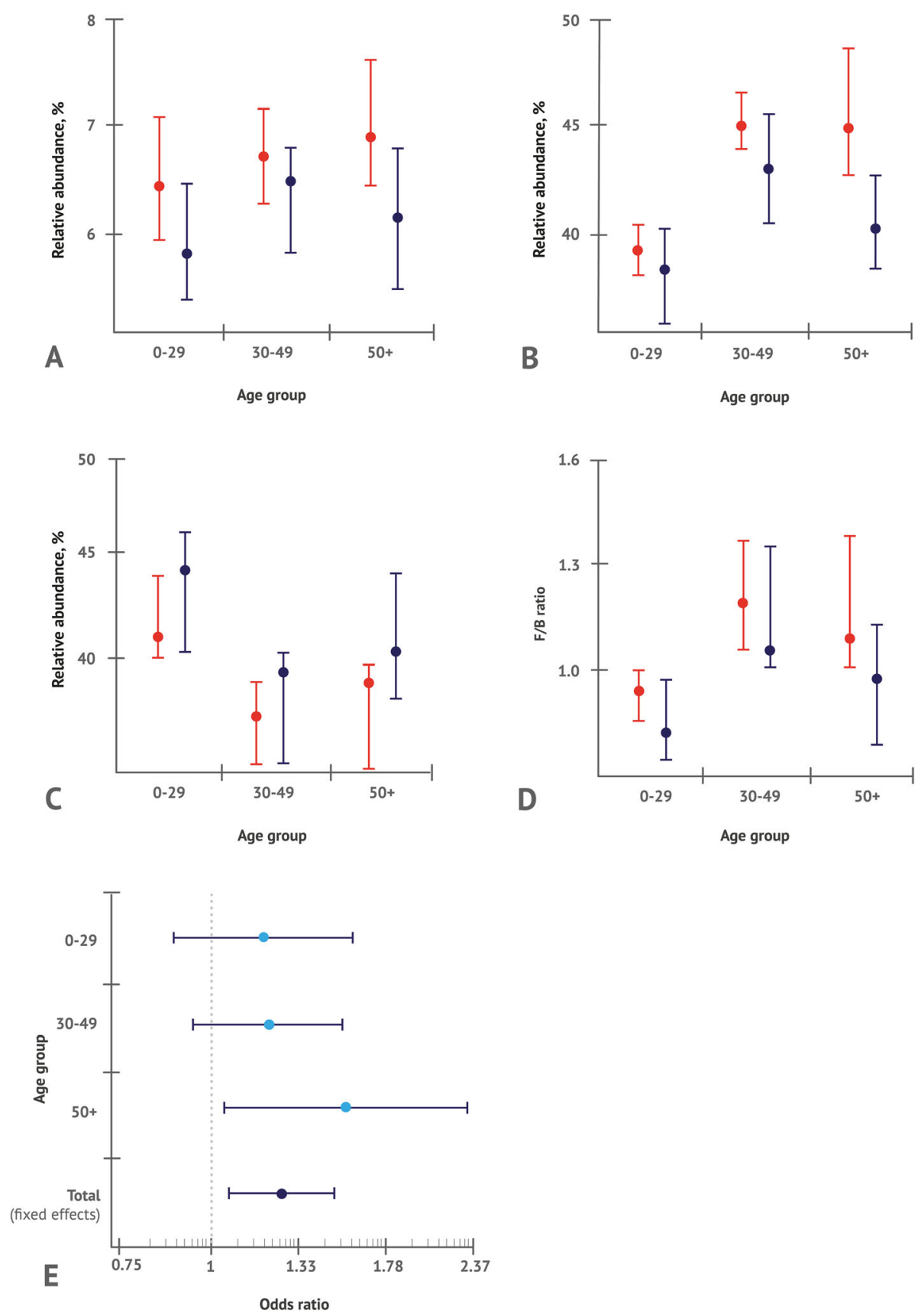

Fig. 3 Relative abundances of major gut microbiota phyla in different age groups: a Firmicutes, b Bacteroidetes, c Actinobacteria, and $\mathbf{d}$ F/B ratio. Data are given as median values (circles) with whiskers indicating $95 \%$ confidence intervals (Cls). e Odds of having F/B ratio $>1$ in women compared to men in different age groups. In the figure, ORs and $95 \% \mathrm{Cls}$ are presented. In all panels, data are expressed as median values (cicles) with whiskers indicating $95 \% \mathrm{Cls}$

approach found that males had three times higher odds than females of having stool community type D, which is generally characterized by lesser levels of Bacteroides and higher of Prevotella than other (A and C) microbial community types [14]. In an Italian population, substantial differences between sexes were observed in a mucosaassociated microbiota (MAM) composition [15]. More specifically, higher abundances of bacterial genera belonging to the Actinobacteria phylum, particularly the genus Bifidobacterium, and a significant depletion in Veillonellaceae were found in female stool samples compared to male ones. Moreover, at the species level, female MAM samples were enriched with Bifidobacterium adolescentis, while male ones were enriched with Gemmiger formicilis [15]. In 
Table 1 Results of two-way ANOVA on the effects of age and sex on abundance of the main bacterial phyla identified in the gut microbiota

\begin{tabular}{|c|c|c|c|}
\hline Source of variation & DF & $F$ & $P$ \\
\hline \multicolumn{4}{|l|}{ Actinobacteria } \\
\hline Sex & 1 & 3.73 & 0.05 \\
\hline Age & 2 & 0.72 & 0.49 \\
\hline Sex×Age Interaction & 2 & 0.21 & 0.81 \\
\hline \multicolumn{4}{|l|}{ Bacteroidetes } \\
\hline Sex & 1 & 3.91 & 0.05 \\
\hline Age & 2 & 16.58 & $<0.001$ \\
\hline Sex×Age Interaction & 2 & 0.31 & 0.74 \\
\hline \multicolumn{4}{|l|}{ Firmiticus } \\
\hline Sex & 1 & 6.27 & 0.01 \\
\hline Age & 2 & 21.40 & $<0.001$ \\
\hline Sex×Age Interaction & 2 & 0.77 & 0.46 \\
\hline \multicolumn{4}{|l|}{ F/B } \\
\hline Sex & 1 & 4.19 & 0.04 \\
\hline Age & 2 & 17.75 & $<0.001$ \\
\hline Sex×Age Interaction & 2 & 0.39 & 0.68 \\
\hline
\end{tabular}

Spain, the abundance of the Bacteroides genus was found to be lower in male than in female individuals, but only when body mass index (BMI) was more than 33 [16]. A higher abundance of Veillonella and Methanobrevibacter in male and Bilophila in female fecal samples was also observed. The abundance of Bilophila was found to be lower in men relative to women. At the bacterial species level, the abundances of Bacteroides caccae was shown to be higher in the female feces, while those of Bacteroides plebeius and Coprococcus catus were higher in male stool samples [16]. In the Netherlands, sex was significantly associated with abundances of 12 microbial species [17]. In particular, female study participants had a higher abundance of Akkermansia muciniphila than the male ones even after controlling for all potentially confounding factors, such as lifestyle, diet and medication. In Japan, significant increases in genera Prevotella, Megamonas, Fusobacterium and Megasphaera were found in the male, and Bifidobacterium, Ruminococcus and Akkermansia in the female fecal samples
[18]. Ruminococcus genus was significantly more abundant in fecal samples from women compared to men in China [19]. In our study, in contrast to these studies, no significant difference was found between sexes for any genus or species tested and for both the intestinal normal flora and conditionally pathogenic flora. These differences in obtained results may be likely attributed to differences in cohort characteristics, in protocols applied, etc.

Phylum-level differences between sexes were reported only in few studies so far. The discovery of sex-specific differences in relative abundance of dominant phyla in the human microbiome such as Bacteroidetes and Firmicutes seems particularly important because the F/B ratio is widely recognized to play an important role in maintaining normal intestinal homeostasis [20]. The reduced number of members of the Bacteroidetes phylum and proportionally elevated number of members of the Firmicutes phylum, known to be associated with a higher capability to supply energy from food, are characteristic features of the "obese gut microbiota" [21].

Data from several studies indicate that relative abundance of Bacteroidetes and Firmicutes can differ between men and women. Higher proportion of the Bacteroidetes phylum was observed in males compared to females in the study by Dominianni et al. [22]. Evidence was obtained that magnitude of these differences may depend on BMI. In the Haro et al. study [16], sexspecific gut microbiome differences were found to be BMI-related, with higher F/B ratio in obese females than that in obese males. More specifically, no differences in $\mathrm{F} / \mathrm{B}$ ratio were revealed between sexes when considered independently of BMI. However, when all study participants were stratified according to BMI, higher F/B ratio was observed in men who had BMI lower than 33 than in women from the same BMI group. By contrast, men had a significantly lower F/B ratio than women in the BMI > 33 group [16]. More recently, a significantly higher relative abundance of Firmicutes in pre-menopausal women compared to their corresponding (age-matched) male control group was found by Santos-Marcos et al. [23]. Findings from several studies are, however, inconsistent and inconclusive. In particular, similar patterns in abundance of Firmicutes and Bacteroidetes have been observed in male and female healthy subjects from 23 populations across four continents [24]. In our research, in contrast with this study and similarly to several

Table $\mathbf{2}$ Logistic regression analysis of the association between sex and F/B ratio

\begin{tabular}{lllll}
\hline Independent variable & Regression coefficient, $\mathbf{b} \pm \mathbf{m}$ & $\boldsymbol{P}$ & OR (95\% Cl) & AUC \\
\hline Sex & & & & $0.53(\mathrm{Cl} 0.51-0.55)$ \\
Male & Reference & 0.002 & $1.31(1.10-1.56)$ & \\
Female & $0.27 \pm 0.09$ & &
\end{tabular}

In Table 3: Event, F/B $>1$, No event, F/B $<1$ 
Table 3 The abundance of the fecal microbial community in study participants at the genus and species levels

\begin{tabular}{|c|c|c|c|c|c|}
\hline \multirow[t]{2}{*}{ Genus/species } & \multicolumn{2}{|l|}{ Female } & \multicolumn{2}{|l|}{ Male } & \multirow[t]{2}{*}{$P^{b}$} \\
\hline & Median $^{a}$ & $95 \% \mathrm{Cl}$ & Median & $95 \% \mathrm{Cl}$ & \\
\hline Akkermansia muciniphilac $^{c}$ & 8.74 & $8.39-8.90$ & 8.48 & $8.00-8.70$ & 0.09 \\
\hline Bacteroides thetaiotaomicron ${ }^{c}$ & 7.85 & $7.70-8.00$ & 7.95 & $7.78-8.00$ & 1.00 \\
\hline Faecalibacterium prausnitzii ${ }^{\mathrm{C}}$ & 9.30 & $9.30-9.48$ & 9.30 & $9.00-9.30$ & 0.42 \\
\hline Clostridium perfringens $^{\mathrm{d}}$ & 6.48 & $6.30-6.60$ & 6.60 & $6.30-6.78$ & 0.42 \\
\hline Fusobacterium nucleatum ${ }^{\mathrm{d}}$ & 5.70 & $5.60-5.85$ & 5.78 & $5.60-5.91$ & 0.45 \\
\hline Parvimonas micra ${ }^{d}$ & 6.15 & $6.00-6.32$ & 6.30 & $6.00-6.30$ & 0.48 \\
\hline Enterobacter spp. and Citrobacter spp. ${ }^{d}$ & 5.00 & $4.78-5.30$ & 5.30 & $4.60-5.48$ & 0.87 \\
\hline
\end{tabular}

${ }^{\mathrm{a}}$ Median values [colony-forming unit (CFU)/cm3] are given in a logarithmic scale in the Table. ${ }^{\mathrm{b}}$ Mann-Whitney test; ${ }^{\mathrm{c}}$ Normal flora; ${ }^{\mathrm{d}}$ Conditionally pathogenic flora

previously reported findings [16, 22-24], relative abundances of major bacterial phyla such as Actinobacteria, Bacteroidetes and Firmicutes, as well as the F/B ratio were found to significantly vary between sexes. Interestingly, this sex difference was even more pronounced in elder individuals (50+) compared to young and adult ones. This is an unexpected result because sex-specific differences are assumed to be affected by the hormonal status, especially in women [6]. Indeed, in the study by Santos-Marcos and colleagues [23], significantly higher relative abundance of Firmicutes was found in pre-menopausal women compared to corresponding (age-matched) male control group, while in post-menopausal women the F/B ratio was found to be similar to those in the corresponding male group. The difference between our findings and those reported by Santos-Marcos and co-authors in the Spanish population [23] can be likely explained by differences in the lifestyle, dietary habits, medications etc., among Spain and Ukraine. These differences may likely affect many important population characteristics, e.g., prevalence of obesity known to be significantly associated with gut microbiota composition. Indeed, the prevalence rate of obesity is approximately equal in adult men and women in Spain (20.2 and 20.9\%, respectively), while in Ukraine this prevalence rate in adult women is almost double of that in men (25.2 and $14.6 \%$, respectively) [25]. Such inter-population difference in prevalence of obesity between sexes may likely be a factor potentially confounding association between the host's sex and the microbiota composition. Unfortunately, we did not have data on weights and heights of the study participants. Therefore, we could not verify the assumption on the impact of BMI on the associations observed. This is the main weakness of our study. Another potential study weakness is unbalanced sample sizes of the female and male groups, with nearly twice as women as men being compared. It is due to the fact that life expectancy is almost ten years less for men than for women in Ukraine (male, 67.6 years; female, 77.1 years in 2016, according to the World Health Statistics 2020 [26]). Therefore, there are much more women than men in the Ukrainian population, especially in older age groups. This can likely explain the larger proportion of women in the sample we studied. Moreover, although the large-size, population-based approach was the strength of our research, the cross-sectional design, which precludes causal inference, was one more limitation of the study. Further, longitudinal designs are certainly needed to draw strong conclusions about causality. Moreover, additional mechanistic investigation is necessary in determining how intestinal microbiota can contribute to sexspecific differences in the initiation and progression of chronic diseases and in developing the microbiota-based therapeutic interventions.

\section{Conclusions}

The main finding of this study is that there are sexspecific differences in the phylum-level gut microbiota composition in individuals from the Ukraine population. In particular, the F/B ratio was found to be significantly increased in females compared to males. Sex-related difference in the microbiota composition is often ignored by researchers. Findings from this and other studies indicate that sex may be a potentially significant factor in determining the microbiome composition. Therefore, this factor must be taken into account in further microbiome research. Further investigation is needed to draw strong conclusions regarding the mechanistic basis for sex-specific differences in the gut microbiota composition and regarding the role of these differences in the initiation and progression of human chronic disorders.

\section{Methods}

\section{Study population}

Fecal samples have been obtained from all the study participants over the period from March 17, 2017 to December 9, 2020 from 2301 relatively healthy individuals (female, 1515; male, 786) residing in Ukraine and visited medical clinics in cities Dnipro and Kyiv for laboratory examination followed by a consultation with a clinician to correct dietary and lifestyle habits. Since 
most persons who visited clinic PB MEDICOM-IN located in Dnipro were those who reside in southeast Ukraine regions and those who visited Molecular Genetic Laboratory DIAGEN located in Kyiv were mainly from northwestern and central Ukraine regions. Thus, this study may be considered as a population-based one. Each study participant signed the informed consent form before enrollment indicating her/his consent to provide a stool sample and to use this sample in additional analyses. Exclusion criteria in the study's protocol were: (a) health problems including current infectious diseases or cancer, cognitive impairments, types 1 diabetes or inadequately controlled type 2 diabetes; (b) current intake of prebiotics, probiotics, antibiotics or immunosuppressants; (c) refusal to provide informed consent. The study was performed according to the Declaration of Helsinki. It has been approved by Ethics Committee of the D.F. Chebotarev State Institute of Gerontology (approval number: 88/16; approval date: 28/12/2016). Basic demographic characteristics of the study population are presented in the Supplementary Table S2.

\section{Sample collection and extraction of DNA}

Fecal samples collected immediately upon defecation have been provided by each study participant in stool containers. These samples have been frozen and stored at $-20{ }^{\circ} \mathrm{C}$ for about a week until the DNA isolation. DNA has been extracted from frozen stool aliquots by the phenol-chloroform method with a protocol provided by Zhang and colleagues [27]. DNA samples were finally eluted in the elution buffer (200 $\mu$ l per each sample). Quantity and quality of the DNA samples were assessed with NanoDrop ND-8000 (Thermo Scientific, USA). Samples containing DNA concentrations less than 50 $\mathrm{ng} / \mathrm{ml}$ and/or an A 260/280 less than 1.8 were subjected to ethanol precipitation to meet standards of quality.

\section{Quantitative identification of the gut microbiota abundance in the feces}

Quantity estimation of Actinobacteria, Firmicutes and Bacteroidetes phyla has been conducted using a realtime thermocycler Rotor-Gene 6000 (QIAGEN, Hilden, Germany) by method described previously [28]. PCR reactions were performed in the following conditions: an initial denaturation for $5 \mathrm{~min}$ at $95{ }^{\circ} \mathrm{C}, 30$ cycles of amplification and a a final elongation at $72{ }^{\circ} \mathrm{C}$ for $5 \mathrm{~min}$. A PCR mixture contained 0.05 units/ $\mu$ l of the Taq polymerase (Sigma Aldrich, St. Louis, MO), $0.2 \mathrm{mM}$ of each dNTP, $0.4 \mu \mathrm{M}$ of each primer, reaction buffer and $\sim 10$ ng of DNA and water to $25 \mu \mathrm{l}$. All PCR reactions were performed in triplicates. Both universal and specific threshold cycles (Cts) registered by the thermocycler were used to quantification of target sequences. $\mathrm{Ct}$ values were further transformed into percentages by the formula provided by Bacchetti De Gregoris and colleagues [29]:

$$
\mathrm{X}=(\text { Eff. Univ })^{\mathrm{Ct}_{\text {univ }}} /(\text { Eff. Spec })^{\mathrm{Ct}_{\text {spec }}} * 100,
$$

where Cts (either specific or universal) represent threshold cycles registered by the thermal cycler. Eff. Univ represents the calculated efficacy of universal primers $(1=0 \%$ and $2=100 \%)$ and Eff. Spec represents the efficacy of the phylum-specific primers. In the equation, $\mathrm{X}$ is the percentage of $16 \mathrm{~S}$ phylum-specific copy number in a sample.

Bacterial quantity estimation at the level of genera and species was performed by the "COLONOFLOR" reagent kit (LLC AlfaLab, Saint-Petersburg, Russian Federation). The abundance of these bacterial groups was measured in colony-forming units per $\mathrm{cm}^{3}\left[(\mathrm{CFU}) / \mathrm{cm}^{3}\right]$ wet feces according to the instructions of the kit manufacturer. This approach provides an opportunity to correlate reported $\mathrm{Ct}$ values with numbers of CFU of bacteria in the sample [30].

\section{Statistical analysis}

Analyses were conducted with a statistical software package MedCalc ${ }^{\oplus}$ Statistical Software version 19.6.3 (MedCalc Software Ltd, Ostend, Belgium). All the studied variables were found to be non-normally distributed $(p<0.01$ for all variables according to the Shapiro-Wilk test). Therefore, data were further analyzed using non-parametric tests. In order to determine the statistical difference among groups, median values were compared with a Mann-Whitney Utest. Logistic regression analysis was applied to evaluate the effects of sex on the F/B ratio. The predictive accuracy of the model has been assessed by AUC, the area under the receiver operating characteristic (ROC) curve. MantelHaenszel method was used to calculate weighted pooled odds ratios (ORs) under the fixed effects model [31]. The interaction between sex and age was evaluated by two-way ANOVA, with age and sex as grouping factors. A rank transformation procedure was used in order to apply ANOVA to the data [32]. Differences were considered significant at $p<0.05$.

\section{Supplementary Information}

The online version contains supplementary material available at https://doi. org/10.1186/s12866-021-02198-y.

Additional file 1: Table S1. Median values of main microbiota phyla in study participants.

Additional file 2: Table S2. Basic characteristics of the study subjects

Acknowledgements

The authors thank the staff of the Institute of Gerontology, PB MEDICOM-IN and Molecular Genetic Laboratory Diagen for their valuable technical assistance. 


\section{Authors' contributions}

$A K, O L$, and $A V$ : conception and design of research. $V M, M R$, and NK: collection of data. AK, AV, OL, and VG: analysis and interpretation of data. AK and $\mathrm{AV}$ : manuscript drafting. AK, MR, and NK: study supervision and administrative support. All authors have read, revised and approved the final draft of the manuscript.

\section{Funding}

No funds have been allocated to this study.

\section{Availability of data and materials}

The dataset generated and analysed during the current study is available from the corresponding author upon the reasonable request.

\section{Declarations}

\section{Ethics approval and consent to participate}

Each study participant signed the informed consent form before enrollment indicating her/his consent to provide a stool sample and to use this sample in additional analyses. The study was performed according to the Declaration of Helsinki. It has been approved by Ethics Committee of the D.F. Chebotarev State Institute of Gerontology (approval number: 88/16; approval date: 28/12/2016).

\section{Consent for publication}

Not applicable.

\section{Competing interests}

The authors declare that they have no competing interests.

\section{Author details}

${ }^{1}$ Molecular Genetic Laboratory Diagen, Kyiv, Ukraine. ${ }^{2}$ Institute of Gerontology, Vyshgorodskaya st. 67, 04114 Kyiv, Ukraine. ${ }^{3}$ Vasyl Stefanyk Precarpathian National University, Ivano-Frankivsk, Ukraine. ${ }^{4}$ Research and Development Institute, Ivano-Frankivsk, Ukraine. ${ }^{5}$ PB MEDICOM-IN, Dnipro, Ukraine. ${ }^{6}$ Bogomolets National Medical University, Kyiv, Ukraine.

Received: 24 January 2021 Accepted: 16 April 2021

Published online: 30 April 2021

\section{References}

1. Rowland I, Gibson G, Heinken A, Scott K, Swann J, Thiele I, Tuohy K. Gut microbiota functions: metabolism of nutrients and other food components. Eur J Nutr. 2018:57:1-24. doi: https://doi.org/10.1007/s00394-017-1445-8.

2. Lee-Sarwar KA, Lasky-Su J, Kelly RS, Litonjua AA, Weiss ST. MetabolomeMicrobiome Crosstalk and Human Disease. Metabolites. 2020;10:181. doi https://doi.org/10.3390/metabo10050181.

3. Rinninella E, Raoul P, Cintoni M, Franceschi F, Miggiano GAD, Gasbarrini A, Mele MC. What is the Healthy Gut Microbiota Composition? A Changing Ecosystem across Age, Environment, Diet, and Diseases. Microorganisms. 2019;7:14. doi: https://doi.org/10.3390/microorganisms7010014.

4. Kim YS, Unno T, Kim BY, Park MS. Sex Differences in Gut Microbiota. World J Mens Health. 2020;38:48-60. doi: https://doi.org/10.5534/wjmh.190009.

5. Neuman H, Debelius JW, Knight R, Koren O. Microbial endocrinology: the interplay between the microbiota and the endocrine system. FEMS Microbiol Rev. 2015;39:509-21. doi: https://doi.org/10.1093/femsre/fuu010.

6. Cross TL, Kasahara K, Rey FE. Sexual dimorphism of cardiometabolic dysfunction: Gut microbiome in the play? Mol Metab. 2018;15:70-81. doi: https://doi.org/10.1016/jmolmet.2018.05.016.

7. Elderman M, de Vos P, Faas M. Role of Microbiota in Sexually Dimorphic Immunity. Front Immunol. 2018;9:1018. doi: https://doi.org/10.3389/fimmu.2 018.01018 .

8. Vemuri R, Sylvia KE, Klein SL, Forster SC, Plebanski M, Eri R, Flanagan KL. The microgenderome revealed: sex differences in bidirectional interactions between the microbiota, hormones, immunity and disease susceptibility. Semin Immunopathol. 2019;41:265-75. doi: https:/doi.org/10.1007/s00281018-0716-7.

9. Santos-Marcos JA, Haro C, Vega-Rojas A, Alcala-Diaz JF, Molina-Abril H, Leon-Acuña A, et al. Sex Differences in the Gut Microbiota as Potential Determinants of Gender Predisposition to Disease. Mol Nutr Food Res. 2019; 63:e1800870. doi: https://doi.org/10.1002/mnfr.201800870.
10. Razavi AC, Potts KS, Kelly TN, Bazzano LA. Sex, gut microbiome, and cardiovascular disease risk. Biol Sex Differ. 2019;10:29. doi: https://doi.org/1 0.1186/s13293-019-0240-z.

11. Jaggar M, Rea K, Spichak S, Dinan TG, Cryan JF. You've got male: Sex and the microbiota-gut-brain axis across the lifespan. Front Neuroendocrinol. 2020;56:100815. doi: https://doi.org/10.1016/j.yfrne.2019.100815.

12. Gomez A, Luckey D, Taneja V. The gut microbiome in autoimmunity: sex matters. Clin Immunol 2015;159:154-62.

13. Vieira AT, Castelo PM, Ribeiro DA, Ferreira CM. Influence of Oral and Gut Microbiota in the Health of Menopausal Women. Front Microbiol. 2017;8: 1884. doi: https://doi.org/10.3389/fmicb.2017.01884.

14. Ding T, Schloss PD. Dynamics and associations of microbial community types across the human body. Nature. 2014;509:357-60. doi: https://doi. org/10.1038/nature13178.

15. Borgo F, Garbossa S, Riva A, Severgnini M, Luigiano C, Benetti A, et al. Body mass index and sex affect diverse microbial niches within the gut. Front Microbiol. 2018;9:213. doi: https://doi.org/10.3389/fmicb.2018.00213.

16. Haro C, Rangel-Zúñiga OA, Alcalá-Díaz JF, Gómez-Delgado F, Pérez-Martínez $P$, Delgado-Lista J, et al. Intestinal microbiota is influenced by gender and body mass index. PLoS One. 2016;11:e0154090. doi: https://doi.org/10.1371/ journal.pone.0154090.

17. Sinha T, Vich Vila A, Garmaeva S, Jankipersadsing SA, Imhann F, Collij V, et al. Analysis of 1135 gut metagenomes identifies sex-specific resistome profiles. Gut Microbes. 2019;10:358-66. doi: https://doi.org/10.1080/194 90976.2018 .1528822$.

18. Takagi T, Naito Y, Inoue R, Kashiwagi S, Uchiyama K, Mizushima K, et al. Differences in gut microbiota associated with age, sex, and stool consistency in healthy Japanese subjects. J Gastroeterol. 2019;54:53-63. doi: https://doi.org/10.1007/s00535-018-1488-5.

19. Gao X, Zhang M, Xue J, Huang J, Zhuang R, Zhou X, et al. Body mass index differences in the gut microbiota are gender specific. Front Microbiol. 2018; 9:1250. doi: https://doi.org/10.3389/fmicb.2018.01250.

20. Indiani CMDSP, Rizzardi KF, Castelo PM, Ferraz LFC, Darrieux M, Parisotto TM. Childhood Obesity and Firmicutes/Bacteroidetes Ratio in the Gut Microbiota: A Systematic Review. Child Obes. 2018;14:501-9. doi: https://doi. org/10.1089/chi.2018.0040.

21. Mitev K, Taleski V. Association between the Gut Microbiota and Obesity. Open Access Maced J Med Sci. 2019;7:2050-6. doi: https://doi.org/10.3889/ oamjims.2019.586.

22. Dominianni C, Sinha R, Goedert JJ, Pei Z, Yang L, Hayes RB, Ahn J. Sex, body mass index, and dietary fiber intake influence the human gut microbiome. PLoS One. 2015;10:e0124599. doi: https://doi.org/10.1371/ journal.pone.0124599.

23. Santos-Marcos JA, Rangel-Zuñiga OA, Jimenez-Lucena R, Quintana-Navarro GM, Garcia-Carpintero S, Malagon MM, et al. Influence of gender and menopausal status on gut microbiota. Maturitas. 2018;116:43-53. doi: https://doi.org/10.1016/j.maturitas.2018.07.008.

24. Suzuki TA, Worobey M. Geographical variation of human gut microbial composition. Biol Lett. 2014;10:20131037. doi: https://doi.org/10.1098/rsbl.2013.

25. Ng M, Fleming T, Robinson M, Thomson B, Graetz N, Margono C, et al. Global, regional, and national prevalence of overweight and obesity in children and adults during 1980-2013: a systemaic analysis for the Global Burden of Disease Study 2013. Lancet. 2014;384:766-81. doi: https://doi. org/10.1016/50140-6736(14)60460-8.

26. World Health Organization. World health statistics 2020: monitoring health for the SDGs, sustainable development goals. World Health Organization. 2020. https://apps.who.int/iris/handle/10665/332070.

27. Zhang BW, Li M, Ma LC, Wei FW. A widely applicable protocol for DNA isolation from fecal samples. Biochem Genet. 2006;44:503-12.

28. Vaiserman A, Romanenko M, Piven L, Moseiko V, Lushchak O, Kryzhanovska $\mathrm{N}$, et al. Differenes in the gut Firmicutes to Bacteroidetes ratio across age groups in healthy Ukrainian population. BMC Microbiol. 2020;20:221. doi: https://doi.org/10.1186/s12866-020-01903-7.

29. Bacchetti De Gregoris T, Aldred N, Clare AS, Burgess JG. Improvement of phylum- and class-specific primers for real-time PCR quantification of bacterial taxa. J Microbiol Methods. 2011;86:351-6. doi: https://doi.org/10.1 016/j.mimet.2011.06.010.

30. Gromova LV, Ermolenko El, Sepp AL, Dmitrieva YV, Alekseeva AS, Lavrenova NS, et al. Gut Digestive Function and Microbiome after Correction of Experimental Dysbiosis in Rats by Indigenous Bifidobacteria. Microorganisms 2021;9:522. https://doi.org/10.3390/microorganisms9030522. 
31. Mantel N, Haenszel W. Statistical aspects of the analysis of data from retrospective studies of disease. J Natl Cancer Inst. 1959;22:719-48.

32. Conover WJ, Iman RL. Rank transformations as a bridge between parametric and nonparametric statistics. American Statistician. 1981;35:124-9. doi: https://doi.org/10.2307/2683975.

\section{Publisher's Note}

Springer Nature remains neutral with regard to jurisdictional claims in published maps and institutional affiliations.

Ready to submit your research? Choose BMC and benefit from:

- fast, convenient online submission

- thorough peer review by experienced researchers in your field

- rapid publication on acceptance

- support for research data, including large and complex data types

- gold Open Access which fosters wider collaboration and increased citations

- maximum visibility for your research: over $100 \mathrm{M}$ website views per year

At BMC, research is always in progress.

Learn more biomedcentral.com/submissions 